\title{
Clinical course, treatment and outcome of Pneumocystis pneumonia in immunocompromised adults: a retrospective analysis over 17 years
}

Julius J. Schmidt ${ }^{1}$, Catherina Lueck², Stefan Ziesing ${ }^{3}$, Matthias Stoll ${ }^{4}$, Hermann Haller$^{1}$, Jens Gottlieb ${ }^{5}$, Matthias Eder ${ }^{2}$, Tobias Welte ${ }^{5}$, Marius M. Hoeper ${ }^{5}$, André Scherag ${ }^{6,7}$ and Sascha David ${ }^{1 *}$

\begin{abstract}
Background: Despite modern intensive care with standardized strategies against acute respiratory distress syndrome (ARDS), Pneumocystis pneumonia (PCP) remains a life-threatening disease with a high mortality rate. Here, we analyzed a large mixed cohort of immunocompromised patients with PCP, with regard to clinical course and treatment, and aimed at identifying predictors of outcome.

Methods: This was a single-center retrospective analysis in a tertiary care institution across 17 years. Diagnosis of PcP required typical clinical features and microbiological confirmation of Pneumocystis jirovecii. Epidemiological, clinical, laboratory and outcome data were collected from patient records.

Results: A total of 52,364 specimens from 7504 patients were sent for microbiological assessment (3653 with clinical suspicion of Pneumocystis pneumonia). PCP was confirmed in 240 patients, about half of them HIV positive (52\%). The remaining subjects were either solid organ transplant recipients (16.3\%) or suffered from malignancy (15.8\%) or autoimmune diseases (11.7\%). Of note, $95 \%$ of patients with PcP were not receiving chemoprophylaxis. Overall in-hospital mortality was 25.4\%, increasing to $58 \%$ if ICU admission was required. Multivariable regression identified lactate dehydrogenase (LDH) as predictor of in-hospital mortality (adjusted OR 1.17 (95\% Cl 1.09-1.27), $p<0.0001$ ). Mortality in LDH quartiles increased from $8 \%$ to $49 \%$, and a cutoff value of $495 \mathrm{U} / \mathrm{L}$ predicted mortality with sensitivity and specificity of $70 \%$. With regard to treatment, $40 \%$ of patients received trimethoprim-sulfamethoxazole at doses that were lower than recommended, and these patients had a higher mortality risk ( $\mathrm{HR} 1.80(95 \% \mathrm{Cl} 1.10-3.44), p=0.02)$.

Conclusions: PcP remains a life-threatening disease among immunocompromised patients. About half of patients with PCP do not have HIV infection. Initial LDH values might serve as a stratifying tool to identify those patients at high risk of death among patients with HIV and without HIV infection.
\end{abstract}

Keywords: Mortality, Transplantation, Lactate dehydrogenase, LDH, HIV

\footnotetext{
* Correspondence: David.sascha@mh-hannover.de

André Scherag and Sascha David contributed equally to this work and are co-last authors.

Julius J Schmidt and Catherina Lueck contributed equally to this work and are co-first authors.

${ }^{1}$ Department of Nephrology and Hypertension, Hannover Medical School, Carl-Neuberg-Strasse 1, 30625 Hannover, Germany

Full list of author information is available at the end of the article
}

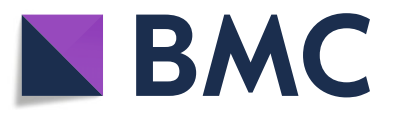

(c) The Author(s). 2018 Open Access This article is distributed under the terms of the Creative Commons Attribution 4.0 International License (http://creativecommons.org/licenses/by/4.0/), which permits unrestricted use, distribution, and reproduction in any medium, provided you give appropriate credit to the original author(s) and the source, provide a link to the Creative Commons license, and indicate if changes were made. The Creative Commons Public Domain Dedication waiver (http://creativecommons.org/publicdomain/zero/1.0/) applies to the data made available in this article, unless otherwise stated. 


\section{Background}

Pneumocystis pneumonia (PcP) is a severe disease with high morbidity and mortality, which almost exclusively affects immunocompromised patients. PcP has long been known for its high prevalence among human immunodeficiency virus (HIV)-positive patients [1]. Since the implementation of combination antiretroviral therapies (cART) and chemoprophylaxis its incidence has been continuously decreasing [2]. Nowadays, most patients with HIV-associated PcP are treatment-naive with very low CD4 cell counts; some of these patients do not know that they are HIV positive until they attend hospital [3]. On the other hand, PcP is also frequently diagnosed in non-HIV-positive patients as immunosuppressive regimens are being increasingly used in a wide range of patient populations. Consistently, the incidence of $\mathrm{PcP}$ in non-HIV-positive patients is increasing [2].

Pneumocystis infections have first been described in preterm infants following World War II [4] and in patients with malignancies in the late 1960s [5], more than a decade before the HIV epidemic emerged. Defects in cell-mediated immunity and use of glucocorticoids are among the strongest risk factors for the development of PcP $[6,7]$.

The epidemiology of PcP has been described in several retrospective studies [2, 8]. A study from the Mayo Clinic in 116 non-HIV-positive patients found that most frequent underlying diseases were hematological malignancies (30\%), organ transplantation (25\%), autoimmune disease (22\%), solid tumors (13\%) and other reasons [6]. Although, most of these reports were published more than a decade ago and might not reflect the current epidemiological situation, a report from France was published a few years ago [9]. Roux et al. reported overall mortality of $17.4 \%$, which was significantly higher in non-HIV-positive patients (27\%) than in HIV-positive patients (4\%) [9]. A few parameters such as age, prior episode of PcP, low CD4 cell count, lactate dehydrogenase (LDH), and coinfections have been reported to predict unfavorable outcome in patients with HIV infection [10-12]. Reports on outcome predictors in non-HIV-positive patients are sparse.

Based on the high burden of PcP and the likelihood of unfavorable outcome particularly in non-HIV-positive patients, chemoprophylaxis with trimethoprim-sulfame thoxazole (TMP-SMX) is recommended in high-risk populations [13]. TMP-SMX is also the treatment of choice for PcP. Adjunctive corticosteroid therapy is recommended in HIV-positive patients with severe respiratory failure $[14,15]$. However, while beneficial outcomes of higher dosage of corticosteroids in HIV-positive patients during PcP are reported [16], the outcome of using corticosteroids in non-HIV-positive patients is not clear [17].

We here report comprehensive epidemiological, clinical, laboratory, therapeutic and outcome data on 240 cases of PcP, including a high percentage of non-HIV-positive patients, in a tertiary care center over the last 17 years. Additionally, we aimed at identifying predictors of outcome.

\section{Methods \\ Study design and population}

We performed a retrospective, single-center cross-sectional analysis of all patients with a positive finding of Pneumocystis jirovecii on direct immunofluorescence testing or detection by Diff-Quick staining in the bronchial washing fluid or broncho-alveolar lavage (BAL) fluid, from January 2000 to June 2017. Our hospital is a university tertiary care center with approximately 1500 beds. Written informed consent was waived by the ethics committee due to the anonymized retrospective nature of the analysis.

All bronchial washing fluids or BAL samples from patients clinically suspected to have $P$. jirovecii pneumonia were evaluated within the study period. For every patient, clinical data on demographic characteristics, underlying disease, status of immune competence, treatment regimens of immunosuppression, PcP therapy regimen and mortality, were gathered in the study database. Date of diagnosis was defined as the date of microbiological confirmation. $P$. jirovecii direct immunofluorescence was performed using the Monofluo kit P. carinii (BioRad Laboratories, years 2000 to 2016) or the Detect IF Pneumocystis carinii kit (Axis-Shield Diagnostics Ltd., year 2017). Diff Quick staining was performed using the stain sets provided by Dade Behring or Siemens AG, respectively.

\section{Statistical analysis}

Standard descriptive statistics were used to summarize the data (e.g. continuous: mean \pm standard deviation/ count: absolute and relative frequencies/time-to-event: Kaplan-Meier estimator). To identify predictors of in-hospital mortality or survival we applied logistic and Cox proportional hazards models. Consequently, both odds ratio (OR) and hazard ratio (HR) estimates were reported. To obtain multivariable adjusted estimates, all main effects with univariate $p$ values less than or equal to 0.15 were investigated simultaneously. In addition, confidence intervals $(\mathrm{CI})$ were calculated with coverage of $95 \%$. Finally, we also created receiver operating characteristics (ROC) curves for in-hospital death, which were summarized by area under the curve (AUC) estimates. ROC analyses were based on an increasingly complex (leave one out) logistic regression model with in-hospital mortality as the predicted outcome and the following predictors, which were all entered linearly: $\mathrm{LDH}$ alone, $\mathrm{LDH}+$ age, $\mathrm{LDH}+$ age + body mass index (BMI), and for $\mathrm{LDH}+$ age + BMI + estimated glomerular filtration rate (eGFR). All reported $p$ values are nominal and two-sided. In this explorative study, we applied a 
significance level of $\alpha=0.05$ (two-sided). All statistical analyses were done using GraphPad Prism 5.0 (La Jolla, CA, USA) or R 3.4.2.

\section{Results \\ Description of study population and etiology of immunosuppression}

During the observation period, 52,364 BAL or bronchial washing fluid samples were investigated. A clinical suspicion of $P$. jirovecii infection was raised in 3652 of these specimens of which 252 (i.e. 6.9\%) were microbiologically confirmed by immunofluorescence as positive for PcP. There were ultimately 240 patients enrolled into the study (12 were excluded due to incomplete data sets) (Fig. 1). In the overall population, 67 patients were female $(27.9 \%)$ and the average age was $45 \pm 15$ years. There were 125 patients (52\%) with HIV infections; 39 (16.3\%) had undergone solid organ transplant and 38 (15.8\%) had undergone chemotherapy due to hematologic or oncologic malignancies: 28 patients (11.7\%) received immunosuppressive therapy for rheumatoid autoimmune diseases (IS/RD) and 10 patients (4.2\%) had other underlying diseases (miscellaneous (MISC)), e. g. common variable immune-deficiency (CVID) (Table 1). Five patients could be classified into multiple categories. After case reevaluation, patients were classified to the most recent active disease prior to the PcP event.

\section{Clinical course of Pneumocystis pneumonia}

The average cumulative incidence of $\mathrm{PCP}$ in our institution was $13 \pm 5$ cases per year, with a peak in the

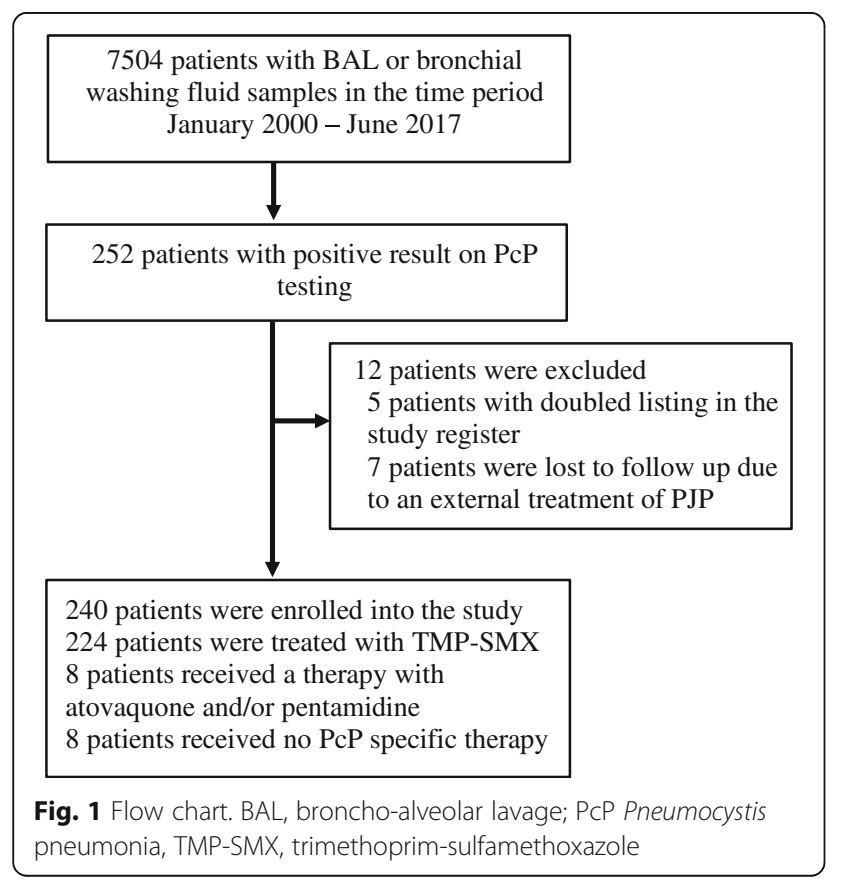

years 2005-2010 (Additional file 1: Figure S1A). Of the 240 patients with PcP, $41.7 \%$ were admitted to the intensive care unit (ICU), with $36.6 \%$ in need of mechanical ventilation, $16.3 \%$ in need of renal replacement therapy (RRT), and $4.5 \%$ in need of extracorporeal membrane oxygenation (ECMO) support (Fig. 2a). The overall in-hospital mortality was $25.4 \%$. The mortality was $58 \%$ in patients requiring ICU treatment, $74.4 \%$ in patients requiring RRT and $81.8 \%$ in patients requiring ECMO support, and only $1.6 \%$ of patients died on regular (non-ICU) wards (Fig. 2b). Mortality during the observation time from 2000 to 2017 slightly fluctuated but did not trend toward an improvement in more recent years (Additional file 1: Figure S1B).

The underlying disease was associated with outcome (Table 1). The lowest mortality was observed in HIV-infected patients (12.8\%); the respective mortality rates in the non-HIV group were $38.4 \%$ in solid organ transplant recipients, $30.0 \%$ in patients with rheumatic diseases and $44.7 \%$ in patients with hematologic -oncologic diseases, respectively (Additional file 1: Figure $\mathrm{S} 1 \mathrm{C}$ ). Figure 2c, d summarizes ICU admission and ICU mortality with regard to the etiology of the patients' immunosuppressive disease. Of note, only 12 patients (5\%) were receiving chemoprophylaxis with TMP-SMX at the time when PcP was diagnosed (Table 1).

\section{Predictors of in-hospital mortality}

Univariate regression analysis revealed that age, BMI, GFR and initial LDH were associated with death from PcP. Both logistic and cox multivariable regression analyses identified $\mathrm{LDH}$ as a predictor of unfavorable outcome in PcP (Table 2, adjusted OR 1.17 (95\% CI 1.091.27) per $50 \mathrm{U} / \mathrm{L}, p<0.0001$, adjusted HR 1.07 (95\% CI $1.04-1.10)$ per $50 \mathrm{U} / \mathrm{L}, p<0.0001)$.

The initial LDH levels were significantly higher in later non-survivors $(443 \pm 214$ vs. $673 \pm 373 \mathrm{iU} / \mathrm{L}, \mathrm{p}<0.0001$, Fig. $3 \mathrm{a})$ and in patients that required admission to the ICU $(411 \pm 199$ vs. $627 \pm 328$ iU/L, $p<0.0001$, Fig. 3b). Stratification of patients into LDH quartiles showed increasing (in-hospital) mortality rates among those. Mortality ranged between $8 \%$ in the lowest and $49 \%$ in the highest LDH quartile (Fig. 3c). A ROC curve for in-hospital death had an estimated AUC of 0.724 (95\% CI $0.65-0.80)(p<0.0001$ for LDH alone, Additional file 1: Figure S2). Potential alternative cutoff values and their impact on usually reported statistics are summarized in Additional file 2: Table S1 for descriptive purposes. We used a cutoff of $496 \mathrm{iU} / \mathrm{L}$ for further analysis. Patients with initial LDH below $496 \mathrm{iU} / \mathrm{L}$ had lower mortality than the overall population (13.1 vs. $25.4 \%$ ) and lower mortality than those with initial LDH > $496 \mathrm{iU} / \mathrm{L}$ (13.1 vs. $43.9 \%, p$ $<0.001$, Fig. 3d, e). 
Table 1 Outcome and characteristics of the investigated study population of patients with PCP

\begin{tabular}{|c|c|c|c|c|c|c|}
\hline & $\begin{array}{l}\text { Overall } \\
240(100 \%) \\
\end{array}$ & $\begin{array}{l}\text { HIV } \\
125 \text { (52\%) }\end{array}$ & $\begin{array}{l}\text { SOT } \\
39(16.3 \%) \\
\end{array}$ & $\begin{array}{l}\text { ChTx } \\
38(15.8 \%) \\
\end{array}$ & $\begin{array}{l}\text { IS/RD } \\
28(11.7 \%) \\
\end{array}$ & $\begin{array}{l}\text { MISC } \\
10(4.2 \%) \\
\end{array}$ \\
\hline Age (years) & $44.8 \pm 14.6$ & $41 \pm 10.6$ & $49.5 \pm 13.9^{* * *}$ & $49.7 \pm 17.2^{* * *}$ & $51.2 \pm 17.6^{* * *}$ & $37.3 \pm 24.5$ \\
\hline Women & $67(27.9 \%)$ & $18(14.4 \%)$ & $11(28.2 \%)$ & $17(44.7 \%)^{\bullet \bullet \bullet}$ & $15(53.6 \%)^{\bullet \bullet \bullet}$ & $6(60 \%)^{* 4}$ \\
\hline ICU admission & $100(41.7 \%)$ & $31(24.8 \%)$ & $19(48.7 \%)^{\bullet \bullet}$ & $27(71.1 \%)^{\bullet \bullet \bullet}$ & $16(57.1 \%)^{\bullet \bullet}$ & $7(70 \%)^{\star \star ~}$ \\
\hline Mechanical ventilation & $88(36.7 \%)$ & $26(22.4 \%)$ & $17(43.6 \%)^{\bullet}$ & $21(55.3 \%)^{\bullet \bullet \bullet}$ & $16(57.1 \%)^{\bullet+4}$ & $6(60 \%)$ \\
\hline CRRT & $39(16.3 \%)$ & $4(3.2 \%)$ & $19(48.7 \%)^{4+4}$ & $6(15.8 \%)^{\bullet}$ & $7(25 \%)^{+4 \bullet}$ & $3(30 \%)^{\star \bullet}$ \\
\hline $\mathrm{ECMO} / \mathrm{ECLA}$ treatment & $11(4.6 \%)$ & $5(4 \%)$ & $3(7.7 \%)$ & $2(5.3 \%)$ & $1(3.6 \%)$ & $0(0 \%)$ \\
\hline in-hospital mortality (\%) & $61(25.4 \%)$ & $16(12.8 \%)$ & $15(38.5 \%)^{\star \star ~}$ & $17(44.7 \%)^{\bullet \bullet \bullet}$ & $10(35.7 \%)^{\bullet \bullet}$ & $3(30 \%)$ \\
\hline TMP-SMX prophylaxis (\%) & $12(5 \%)$ & $6(4.8 \%)$ & $1(2.6 \%)$ & $5(13.2 \%)$ & $0(0 \%)$ & $0(0 \%)$ \\
\hline initial LDH (U/L) & $502.1 \pm 281.6$ & $482.2 \pm 247.2$ & $482.5 \pm 374.0$ & $498.0 \pm 236.4$ & $627.3 \pm 347.2^{*}$ & $493.5 \pm 160.5$ \\
\hline
\end{tabular}

Abbreviations: PCP Pneumocystis pneumonia, ICU intensive care unit, CRRT continuous renal replacement therapy, ECMO extracorporeal membrane oxygenation, ECLA extracorporeal lung assist, TMP-SMX trimethoprim-sulfamethoxazole, HIV human immunodeficiency virus, SOT solid organ transplantation,

ChTx chemotherapy, IS/RD immunosuppression/rheumatic diseases. MISC miscellaneous

Difference compared to HIV-positive patients (Fisher's exact test): ${ }^{*} p<0.05,{ }^{*} p<0.01,{ }^{*} " p<0.001$

Difference compared to HIV-positive patients (Mann-Whitney U test): $p<0.05,{ }^{* *} p<0.01,{ }^{* * *} p<0.001$
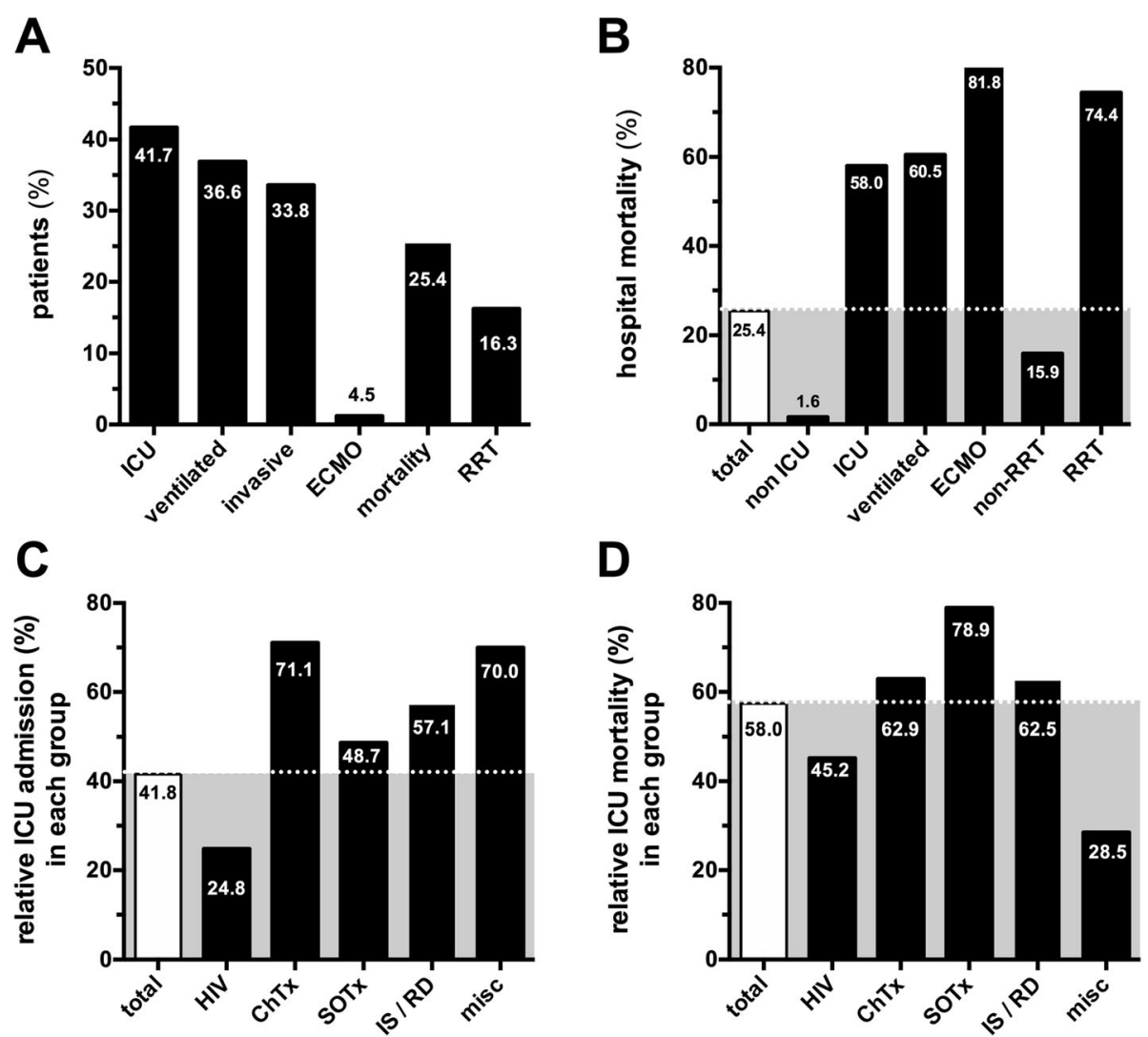

Fig. 2 Etiology and clinical course of Pneumocystis pneumonia (PCP). a Percentage of patients with respect to clinical course of PcP including organ replacement therapies (ICU, intensive care unit; ECMO, extracorporeal membrane oxygenation; RRT, renal replacement therapies) and outcomes. b Hospital mortality in different clinical settings (gray area highlights overall mortality). c Percentage of ICU admission and $\mathbf{d}$ percentage of ICU mortality with immunosuppression with different etiologies. The gray area shows the total percentage independent from the etiology. SOT, solid organ transplant; ChTx, chemotherapy; IS/RD, immunosuppression/rheumatic disease 


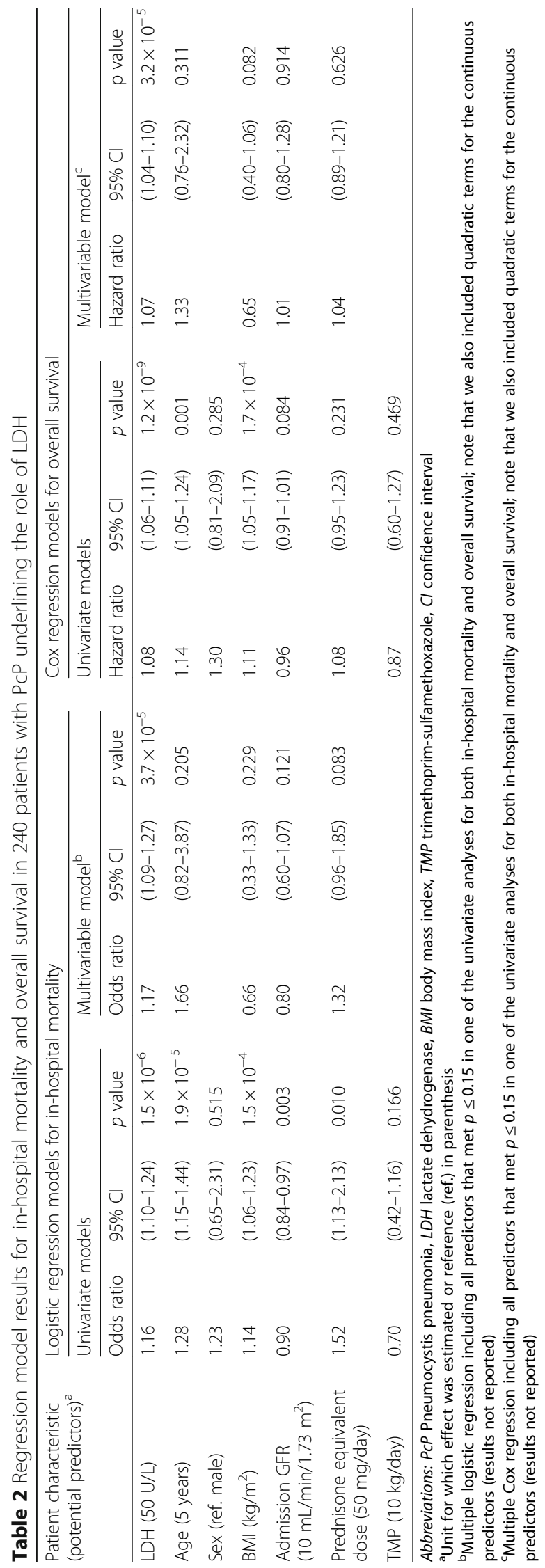



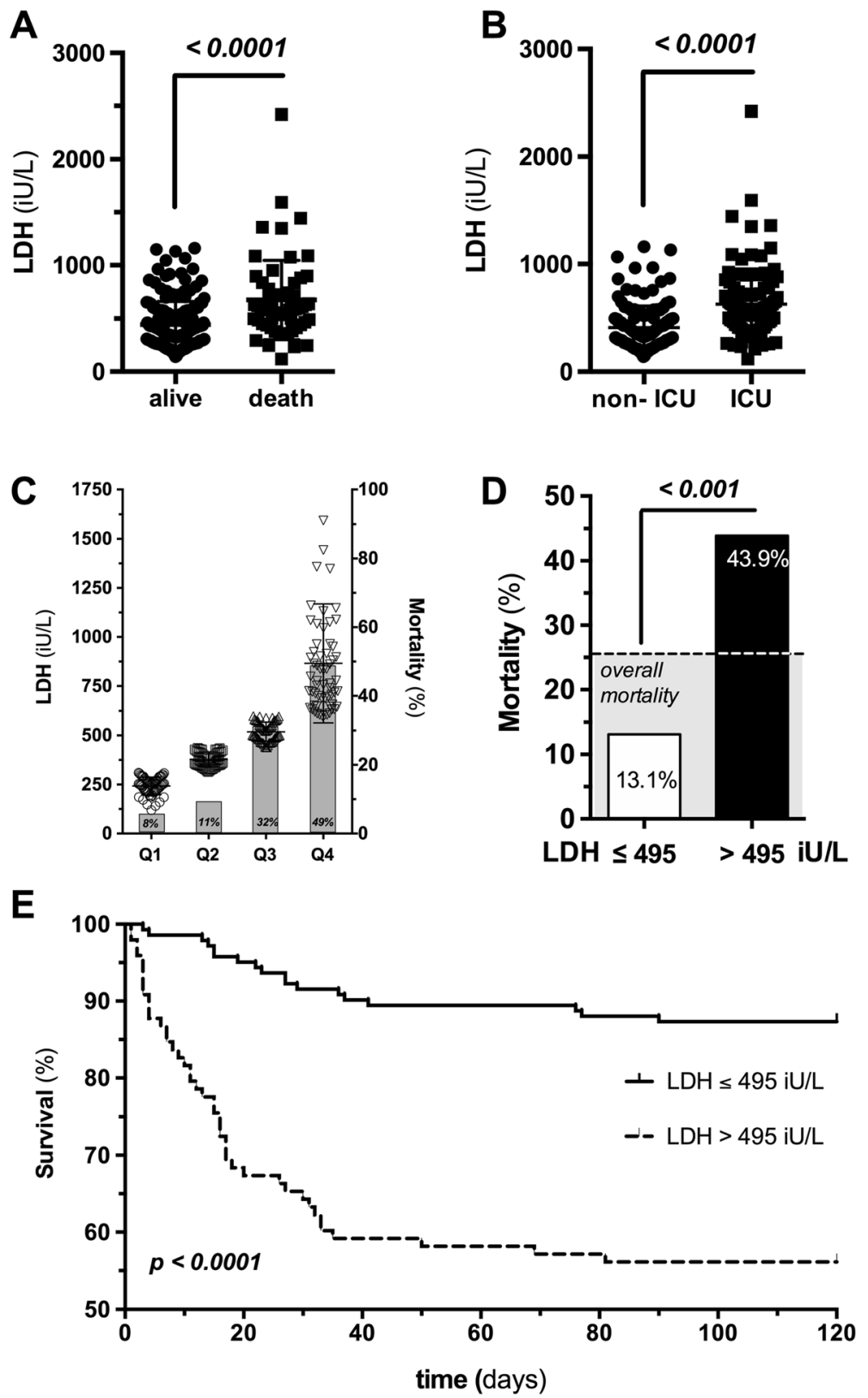

Fig. 3 Influence of initial lactate dehydrogenase (LDH) as an outcome predictor. The scatter plot shows initial LDH levels at admission in later survivors (alive) and non-survivors (dead) $(443 \pm 214$ vs. $673 \pm 373 \mathrm{iU} / \mathrm{L}, p<0.0001)$ (a) and in non-ICU versus ICU patients $(411 \pm 199$ vs. $627 \pm 328$ $\mathrm{iU} / \mathrm{L}, p<0.0001)(\mathbf{b})$. c Bar graph showing overall mortality (right y-axis) stratified for initial LDH quartiles (left y-axis) (Q1, <310; Q2, 311-436; Q3, 437-599; Q4, > $600 \mathrm{iU} / \mathrm{L}$ ). d Mortality in all patients with Pneumocystis pneumonia with LDH levels $\leq$ and $>496$ iU/L (cutoff value was derived from ROC curve of all patients, with sensitivity and specificity of 70\%.) The gray area highlights the overall mortality of $24.8 \%$ without LDH risk stratification. e Kaplan-Meier analysis of survival in patients stratified for LDH $\leq$ and $>495 \mathrm{iU} / \mathrm{L}$ during 120 days $(p$ log-rank test $<0.0001)$

The addition of other clinical quantitative variables to LDH in the AUC model such as age, BMI and eGFR did not improve the predictive value of LDH alone (Additional file 1: Figure S2, Additional file 2: Table S2) in the overall cohort. However, when ICU patients were analyzed separately, we observed that the addition of age as a variable increased the predictive performance of $\mathrm{LDH}$ alone from an

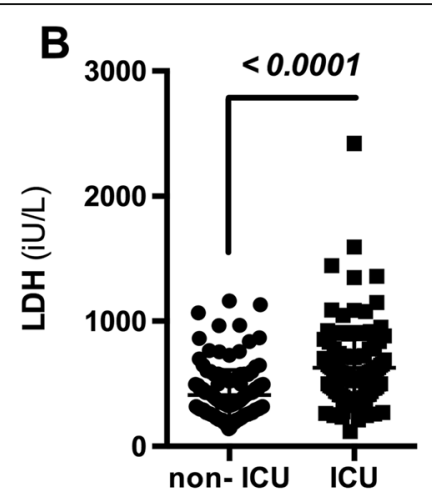

AUC of 0.61 (95\% CI 0.49-0.72) to an AUC of 0.71 (95\% CI $0.60-0.81)(p=0.024)$. Of note, we did not detect any etiology subgroup that showed better performance in the ROC analysis (Additional file 1: Figure S3), nor did we detect temporal trends when applying the $\mathrm{LDH}$ prediction models in three strata (2000-2005, 2005-2010, 2010-2017) (Additional file 1: Figure S4). 


\section{Treatment patterns and outcome}

There were 224/240 patients (93.3\%) initially treated with the gold standard, TMP-SMX. The remaining 16 patients had alternative regimens with chemotherapeutics from the second line, mostly due to side effects and intolerance of TMP/SMX (e.g. atovaquone and/or pentamidine, Fig. 1). Among the 224 patients treated with TMP-SMX, 159 (71\%) were dosed in the recommended range above $15 \mathrm{mg} / \mathrm{kg}$ bodyweight (BW) per day, while 65 patients (29\%) received a dose lower than $15 \mathrm{mg} / \mathrm{kg}$ BW (Additional file 1: Figure S5A). Of note, these patients had a higher mortality risk (HR 1.80 (95\% CI $1.10-3.44), p=0.02$ ). Solid organ transplant (SOT) recipients and patients with rheumatoid diseases were more likely to receive lower dosages (Additional file 1: Figure S5B). On the other side, we observed that under-dosed patients also had significantly lower eGFR $(48.9 \pm 43 \mathrm{ml} / \mathrm{min}$ vs. $104 \pm$ $32 \mathrm{ml} / \mathrm{min}, p<0.0001)$. Interestingly, the absolute dose of TMP-SMX was not different in survivors $(16.9 \pm 5.5 \mathrm{mg} / \mathrm{kg})$ and non-survivors $(15.6 \pm 7.1 \mathrm{mg} / \mathrm{kg}, \quad p=0.93)$. Still, Kaplan-Meier survival curves revealed a significant difference when patients were grouped into those treated with $<15 \mathrm{mg} / \mathrm{kg}$ and those treated with $\geq 15 \mathrm{mg} / \mathrm{kg}$ TMP-SMX (Additional file 1: Figure $\mathrm{S} 5 \mathrm{C}, p_{\text {log-rang test }}=0.02$ ). However, TMP-SMX dose did not independently predict outcome in the multivariable regression analyses.

\section{Discussion}

Here we present the single-center experience with regard to epidemiology, treatment and outcome of PcP in a tertiary care center over a period of 17 years. A substantial group of our PcP cases (about 50\%) were not related to HIV infections and these patients had a worse clinical course with higher ICU admission and mortality rates than patients with HIV-associated PcP. Three major non-HIV-positive groups were identified: (1) solid organ transplant recipients, (2) patients with malignancies and (3) patients with rheumatic diseases. PcP occurred almost exclusively in patients who did not receive chemoprophylaxis with TMP-SMX. Overall, about one quarter of all patients suffering from PcP did not survive the disease. In those who required intensive care (approximately $40 \%$ of all patients), the in-hospital mortality increased up to $58 \%$. Based on the - in principle - reversible nature of PcP, almost no patients were denied intensive care in the event of acute respiratory failure, as the in-hospital mortality rate in non-ICU patients was $1.6 \%$. Moreover, we found that the extent of initial LDH elevation was a predictor of in-hospital mortality, and that in univariate comparisons, in-hospital mortality was higher in patients whose TMP-SMX dose was below $15 \mathrm{mg} / \mathrm{kg} \mathrm{BW}$.

Non-HIV-positive populations at risk of PcP have been described before. The distribution across these risk groups varies widely (SOT 3-31\%, hematologic diseases
$26-39 \%$, rheumatic diseases $11-25 \%$ ), which is likely due to the clinical emphasis in predominantly monocentric studies $[9,18-20]$. Several centers have reported a decrease in the percentage of HIV-infected patients among all cases of PcP in the era of cART $[19,21]$. We did not confirm this decrease, nor did Fillatre et al. [18].

Our data showed that the clinical course and outcome of PcP differed between HIV-infected and non-HIV-infected patients. In general, HIV-infected patients require fewer ICU admissions and have better overall survival than non-HIV-infected patients, which is in line with previous reports $[9,22]$. The PcP mortality rate in HIV-positive patients was in the range of $1-15 \%[9,18,19]$ compared to $30-40 \%$ in HIV-negative patients [23-25]. The better outcome of HIV-positive patients might be the consequence of (1) lower age (-3.8 years in our cohort), (2) fewer co-morbidities, (3) the reversible nature of the immune defect upon anti-viral treatment strategies and hypothetically (4) greater awareness of PcP in HIV-positive individuals, where it is the most common AIDS-defining disease.

In mixed cohorts, ICU admission and mechanical ventilation occurred in $22-40 \%$ and $13-22 \%$ of patients, respectively $[9,18,22]$. Both events are frequently reported as negative predictors of survival. Nevertheless, newer data on ICU mortality in patients with PcP are scarce. Only one recent study reported relatively high ICU mortality in HIV-negative patients of $53 \%$ compared to $15 \%$ in HIV-positive patients [18]. Mortality in patients with invasive mechanical ventilation was $60 \%$ and $28 \%$, respectively, in these two groups [22]. Compared to other reports $[21,26]$, we had a relatively high ICU admission rate of $25 \%$ in HIV-positive patients, which can in part be explained by the selective transfer of more patients with more progressive disease to our university hospital as a tertiary center.

The literature is inconclusive on outcome predictors, but on multivariable analysis, a large prospective study from France demonstrated a significant survival benefit in younger patients [9]. Although observed in HIV-positive patients before [27], here we report for the first time that in a mixed population of HIV-positive and non-HIV-positive patients the initial levels of serum LDH at hospital admission are not only useful in the diagnostic evaluation [28] but may also be an independent predictor of survival. This might potentially help the clinician in early identification of the sickest patients at high risk of unfavorable outcome. With regard to the specificity/sensitivity, the ideal LDH cutoff value to predict outcome has yet to be defined.

With regard to the actual treatment of PcP we found that a reduction in the recommended TMP-SMX dose below $15 \mathrm{mg} / \mathrm{kg} \mathrm{BW}$ (for whatever reason) might be associated with higher mortality (13.1 vs. $55.8 \%$ ). Given that this observation was not confirmed in the multivariable analysis, relevant confounders must be considered. 
Our study has several limitations. This was a retrospective observational study based on the medical records of patients with PcP from a single institution. Consequently, causal claims cannot be made. The observation that under-dosing with TMP-SMX is associated with higher mortality might be confounded by a higher percentage of patients with renal impairment. More robust evidence from well-designed studies is needed to make firm conclusions or even to make implications about changes in standard PcP management.

\section{Conclusions}

In summary, $\mathrm{PcP}$ is a rare but potentially fatal disease in immunocompromised patients with diseases of different etiology. About $50 \%$ of cases were non-HIV-associated. Initial LDH levels - if validated by others - might be a useful predictor of in-hospital mortality, and TMP-SMX treatment doses in patients at high risk of death (e.g. ICU admission + LDH $>495 \mathrm{U} / \mathrm{L}$ ) should probably not be reduced below $15 \mathrm{mg} / \mathrm{kg} \mathrm{BW}$.

\section{Additional files}

Additional file 1: Figure S1. Cumulative incidence of (A) and inhospital mortality in (B) Pneumocystis pneumonia (PCP) at Hannover Medical School from 2000 to 2017. Figure S2. Receiver operating characteristic (ROC) curves for in-hospital mortality applied to the total sample. Figure S3. ROC curves for in-hospital mortality applied to subgroups regarding underlying etiology of immunosuppression and for the ICU COhort. Figure S4. ROC curves for in-hospital mortality with the LDH prediction model applied in three strata (years 2000-2005, 2005-2010, 2010-2017). Figure S5. Association between trimethoprimsulfamethoxazole (TMP-SMX) dose and mortality (DOCX $21 \mathrm{~kb}$ )

Additional file 2: Table S1. Suggested, alternative data-derived LDH cutoff values and their impact on usually reported statistics (with 95\% confidence interval in parenthesis) related to the prediction of in-hospital mortality in patients with PCP. Table S2. Estimated AUCs (with 95\% confidence interval) of the LDH-related logistic regression models for the prediction of in-hospital mortality in patients with PCP. Table S3. Additional descriptive information (with 95\% confidence interval in parenthesis) on patient characteristics and their potential value as predictors of in-hospital mortality in patients with PCP. Table S4. Estimated AUCs (with 95\% confidence interval) of the LDH-related logistic regression models for the prediction of in-hospital mortality in patients with PCP. (DOCX $10367 \mathrm{~kb}$ )

\footnotetext{
Abbreviations

AUC: Area under the curve; BAL: Broncho-alveolar lavage; BMI: Body mass index; BW: Bodyweight; CART: Combination antiretroviral therapies; $\mathrm{Cl}$ : Confidence interval; CRRT: Continuous renal replacement therapy; CVID: Common variable immune-deficiency; ECMO: Extracorporeal membrane oxygenation; eGFR: Estimated glomerular filtration rate; HIV: Human immunodeficiency virus; HR: Hazard ratio; IS: Immunosuppressive; LDH: Lactate dehydrogenase; OR: Odds ratio; PCP: Pneumocystis pneumonia; RD: Rheumatic disease; ROC: Receiver operating characteristic curve; SOT: Solid organ transplantation; TMPSMX: Trimethoprim-sulfamethoxazole
}

\section{Acknowledgments}

none

\section{Funding}

No external funding was received to conduct this study. However, Dr David's laboratory is supported by a grant from the German Research Foundation (DA1209/4-3).

\section{Availability of data and materials}

The datasets used and analyzed during the current study are available from the corresponding author on reasonable request.

\section{Authors' contributions}

JJS, CL, SD and SZ collected the data. AS performed the statistical analysis. Experts in particular field were involved for specific data interpretation (MS for HIV; HH, JG, TB, MMH for transplantation; ME for malignancies). JJS, CL, $\mathrm{MMH}, \mathrm{AS}$ and SD wrote the manuscript and all authors read, commented on and approved it.

\section{Ethics approval and consent to participate}

Written informed consent was waived by the local ethics committee at Hannover Medical School due to the anonymized retrospective nature of the analysis.

\section{Consent for publication}

Not applicable.

\section{Competing interests}

The authors declare that they have no competing interests.

\section{Publisher's Note}

Springer Nature remains neutral with regard to jurisdictional claims in published maps and institutional affiliations.

\section{Author details}

${ }^{1}$ Department of Nephrology and Hypertension, Hannover Medical School, Carl-Neuberg-Strasse 1, 30625 Hannover, Germany. 'Department of Hematology, Hemostasis, Oncology and Stem Cell Transplantation, Hannover Medical School, Hannover, Germany. ${ }^{3}$ Department of Microbiology, Hannover Medical School, Hannover, Germany. ${ }^{4}$ Department of Clinical Immunology and Rheumatology, Hannover Medical School, Hannover, Germany. ${ }^{5}$ Department of Pneumology, Hannover Medical School, and German Center for Lung Research (DZL), Hannover, Germany. ${ }^{6}$ Institute of Medical Statistics, Computer and Data Sciences, Jena University Hospital, Jena, Germany. ${ }^{7}$ Center for Sepsis Control and Care, Jena University Hospital, Jena, Germany.

Received: 6 June 2018 Accepted: 5 October 2018

Published online: 19 November 2018

References

1. Johnson NM. Pneumonia in the acquired immune deficiency syndrome. $\mathrm{Br}$ Med J (Clin Res Ed). 1985;290:1299-301.

2. Sepkowitz KA. Opportunistic infections in patients with and patients without acquired immunodeficiency syndrome. Clin Infect Dis. 2002;34: 1098-107.

3. Fei MW, Sant CA, Kim EJ, Swartzman A, Davis JL, Jarlsberg LG, et al. Severity and outcomes of Pneumocystis pneumonia in patients newly diagnosed with HIV infection: an observational cohort study. Scand J Infect Dis. 2009; 41:672-8.

4. Vanek J, Jirovec O. Parasitic pneumonia. Interstitial plasma cell pneumonia of premature, caused by Pneumocystis carinii. Zentralbl Bakteriol Parasitenkd Infektionskr Hyg. 1952;158:120-7.

5. Walzer PD, Perl DP, Krogstad DJ, Rawson PG, Schultz MG. Pneumocystis carinii pneumonia in the United States. Epidemiologic, diagnostic, and clinical features. Ann Intern Med. 1974;80:83-93.

6. Yale SH, Limper AH. Pneumocystis carinii pneumonia in patients without acquired immunodeficiency syndrome: associated illness and prior corticosteroid therapy. Mayo Clin Proc. 1996;71:5-13.

7. Sepkowitz KA, Brown AE, Telzak EE, Gottlieb S, Armstrong D. Pneumocystis carinii pneumonia among patients without AIDS at a cancer hospital. JAMA. 1992;267:832-7.

8. Jarboui MA, Mseddi F, Sellami H, Sellami A, Makni F, Ayadi A. Pneumocystis: epidemiology and molecular approaches. Pathol Biol. 2013;61:239-44. 
9. Roux A, Canet E, Valade S, Gangneux-Robert F, Hamane S, Lafabrie A, et al Pneumocystis jirovecii pneumonia in patients with or without AIDS, France. Emerging Infect Dis. 2014;20:1490-7.

10. Antinori A, Maiuro G, Pallavicini F, Valente F, Ventura G, Marasca G, et al. Prognostic factors of early fatal outcome and long-term survival in patients with Pneumocystis carinii pneumonia and acquired immunodeficiency syndrome. Eur J Epidemiol. 1993;9:183-9.

11. Fernandez P, Torres A, Miro JM, Vieigas C, Mallolas J, Zamora L, et al. Prognostic factors influencing the outcome in pneumocystis carinii pneumonia in patients with AIDS. Thorax. 1995;50:668-71.

12. Dworkin MS, Hanson DL, Navin TR. Survival of patients with AIDS, after diagnosis of Pneumocystis carinii pneumonia, in the United States. J Infect Dis. 2001;183:1409-12.

13. Iriart X, Challan Belval T, Fillaux J, Esposito L, Lavergne R-A, CardeauDesangles I, et al. Risk factors of Pneumocystis pneumonia in solid organ recipients in the era of the common use of posttransplantation prophylaxis. Am J Transplant. 2015;15:190-9.

14. Walmsley S, Levinton C, Brunton J, Muradali D, Rappaport D, Bast M, et al. A multicenter randomized double-blind placebo-controlled trial of adjunctive corticosteroids in the treatment of Pneumocystis carinii pneumonia complicating the acquired immune deficiency syndrome. J Acquir Immune Defic Syndr Hum Retrovirol. 1995;8:348-57.

15. Montaner JS, Russell JA, Lawson L, Ruedy J. Acute respiratory failure secondary to Pneumocystis carinii pneumonia in the acquired immunodeficiency syndrome. a potential role for systemic corticosteroids. Chest. 1989;95:881-4.

16. Ewald $\mathrm{H}$, Raatz $\mathrm{H}$, Boscacci R, Furrer $\mathrm{H}$, Bucher $\mathrm{HC}$, Briel M. Adjunctive corticosteroids for Pneumocystis jiroveci pneumonia in patients with HIV infection. Cochrane HIV/AIDS Group. Cochrane Database Syst Rev. 2015;323: CD006150.

17. Pareja JG, Garland R, Koziel H. Use of adjunctive corticosteroids in severe adult non-HIV Pneumocystis carinii pneumonia. Chest. 1998;113:1215-24.

18. Fillatre P, Decaux O, Jouneau S, Revest M, Gacouin A, Robert-Gangneux F, et al. Incidence of Pneumocystis jiroveci pneumonia among groups at risk in HIV-negative patients. Am J Med. 2014;127:1242.e11-7.

19. Bienvenu A-L, Traore K, Plekhanova I, Bouchrik M, Bossard C, Picot S. Pneumocystis pneumonia suspected cases in 604 non-HIV and HIV patients. Int J Infect Dis. 2016:46:11-7.

20. Ricciardi A, Gentilotti E, Coppola L, Maffongelli G, Cerva C, Malagnino V, et al. Infectious disease ward admission positively influences $P$. jiroveci pneumonia (PjP) outcome: a retrospective analysis of 116 HIV-positive and HIV-negative immunocompromised patients. Schildgen O. PLoS One. 2017 12:e0176881.

21. Bitar D, Lortholary O, Le Strat $Y$, Nicolau J, Coignard B, Tattevin P, et al. Population-based analysis of invasive fungal infections, France, 2001-2010. Emerging Infect. Dis. 2014;20:1149-55.

22. Guo F, Chen Y, Yang S-L, Xia H, Li X-W, Tong Z-H. Pneumocystis pneumonia in HIV-infected and immunocompromised non-HIV infected patients: a retrospective study of two centers in China. PLoS One. 2014;9:e101943 Santin $M$, editor.

23. Yu Q, Jia P, Su L, Zhao H, Que C. Outcomes and prognostic factors of nonHIV patients with pneumocystis jirovecii pneumonia and pulmonary CMV co-infection: a retrospective cohort study. BMC Infect Dis. 2017:17:392 4 ed.

24. Kofteridis DP, Valachis A, Velegraki M, Antoniou M, Christofaki M, Vrentzos $G E$, et al. Predisposing factors, clinical characteristics and outcome of Pneumonocystis jirovecii pneumonia in HIV-negative patients. J Infect Chemother. 2014;20:412-6.

25. Roblot F, Godet C, Le Moal G, Garo B, Faouzi Souala M, Dary M, et al. Analysis of underlying diseases and prognosis factors associated with Pneumocystis carinii pneumonia in immunocompromised HIV-negative patients. Eur J Clin Microbiol Infect Dis. 2002;21:523-31.

26. Rosen MJ, Clayton K, Schneider RF, Fulkerson W, Rao AV, Stansell J, et al. Intensive care of patients with HIV infection: utilization, critical illnesses, and outcomes. Pulmonary Complications of HIV Infection Study Group. Am J Respir Crit Care Med. 1997;155:67-71.

27. Sun J, Su J, Xie Y, Yin MT, Huang Y, Xu L, et al. Plasma IL-6/IL-10 ratio and $\mathrm{IL}-8, \mathrm{LDH}$, and $\mathrm{HBDH}$ level predict the severity and the risk of death in AIDS patients with Pneumocystis pneumonia. J Immunol Res. 2016;2016: 1583951-10.
28. Boldt MJ, Bai TR. Utility of lactate dehydrogenase vs radiographic severity in the differential diagnosis of Pneumocystis carinii pneumonia. Chest. 1997; 111:1187-92.

\section{Ready to submit your research? Choose BMC and benefit from:}

- fast, convenient online submission

- thorough peer review by experienced researchers in your field

- rapid publication on acceptance

- support for research data, including large and complex data types

- gold Open Access which fosters wider collaboration and increased citations

- maximum visibility for your research: over $100 \mathrm{M}$ website views per year

At BMC, research is always in progress.

Learn more biomedcentral.com/submissions 